\title{
Single Crystal X-ray Diffraction
}

\author{
Virginia B. Pett \\ The College of Wooster \\ Wooster, OH 44691
}




\section{The solid phase}

- Gas molecules have the highest entropy because they have the most degrees of freedom: vibration, rotation and translation

- In liquids molecules vibrate but have limited translation and rotation

- Molecules, atoms or ions in the solid phase have the fewest degrees of freedom

" vibration of bonds (stretching)

"vibration about an equilibrium position

» no rotation or translation 


\section{Most solids have an orderly structure}

- The beautiful external form of a crystal is a sign of the internal orderliness

"flat faces

" sharp edges

" characteristic angles

"Cleavage

"Extinguish polarized light

- In a crystalline solid, atoms, ions or molecules are arranged in a regular, repeating pattern

- An orderly solid will diffract $X$ rays 


\section{Structure $\Rightarrow$ properties}

- Often, solids are composed of microscopic crystallites rather than one large "single" crystal

" a snowflake with hexagonal symmetry

" "feathers" of frost on the windowpane

- Sometimes there are "defects" in the regularity of the internal order-these defects may change the color or electrical properties of the solid

" $\mathrm{Al}_{2} \mathrm{O}_{3}$ with $\mathrm{Cr}^{3+}$ defects has the intense red color of ruby

"In the ruby laser, the $\mathrm{Cr}^{3+}$ ions are excited by light, and lase at $694 \mathrm{~nm}$ 
- Metals and ceramics are crystalline solids but they are often not single crystals; properties depend upon

" structure within crystallites or "grains"

" size of grains

" forces between grains

- Humans have been attempting to alter the parameters of crystalline solids since the bronze age in order to obtain needed properties such as strength, hardness, flexibility, conductivity

- X-ray diffraction is a tool for learning about the structure of solids, even if there aren't single crystals 


\section{The structure of a molecule. . .}

- Often determines its stability and the mechanism by which it will react

- There are many examples from organic chemistry

" reactivity of $\mathrm{C}$ radicals: primary $>\mathrm{sec}>$ tert

" $\mathrm{pK}_{\mathrm{a}}$ of acids depends upon electron-withdrawing and electron-donating groups

"substitution of groups on aromatic rings depends upon position

" $S_{N} 1$ or $S_{N} 2$ mechanism influenced by structure 


\section{Biological structures}

- Are clearly related to function

" enzyme catalyzes a specific reaction

$\checkmark$ the active site accommodates a limited set of reactants

$\checkmark$ the active site facilitates a particular reaction and discourages side reactions

" antibodies protect us from foreign organisms

$\checkmark$ by recognizing the detailed structure of characteristic molecules from bacteria and viruses 


\section{How do we know that benzene and other aromatic molecules are planar?}

- Kathleen Lonsdale determined the structure of hexamethyl benzene by X-ray diffraction in 1928

»X-ray camera

» Film diffraction data

"Trigonometric tables 


\section{How do we know the geometry of nucleophilic substitution?}

- The pathways of chemical reactions have been studied by Jack Dunitz, Hans Bürgi, and others at ETH in Zurich

- In 1973 they detailed the geometric progress of a nucleophilic addition to a carbonyl group by an amino group. They analyzed distances and geometry in many crystal structures, each representing a "snapshot" along the reaction coordinate

- In 1975 they showed by a similar method that a tetrahedral group forms a planar intermediate in an $S_{N} 1$ reaction 


\section{ORTEP diagram}

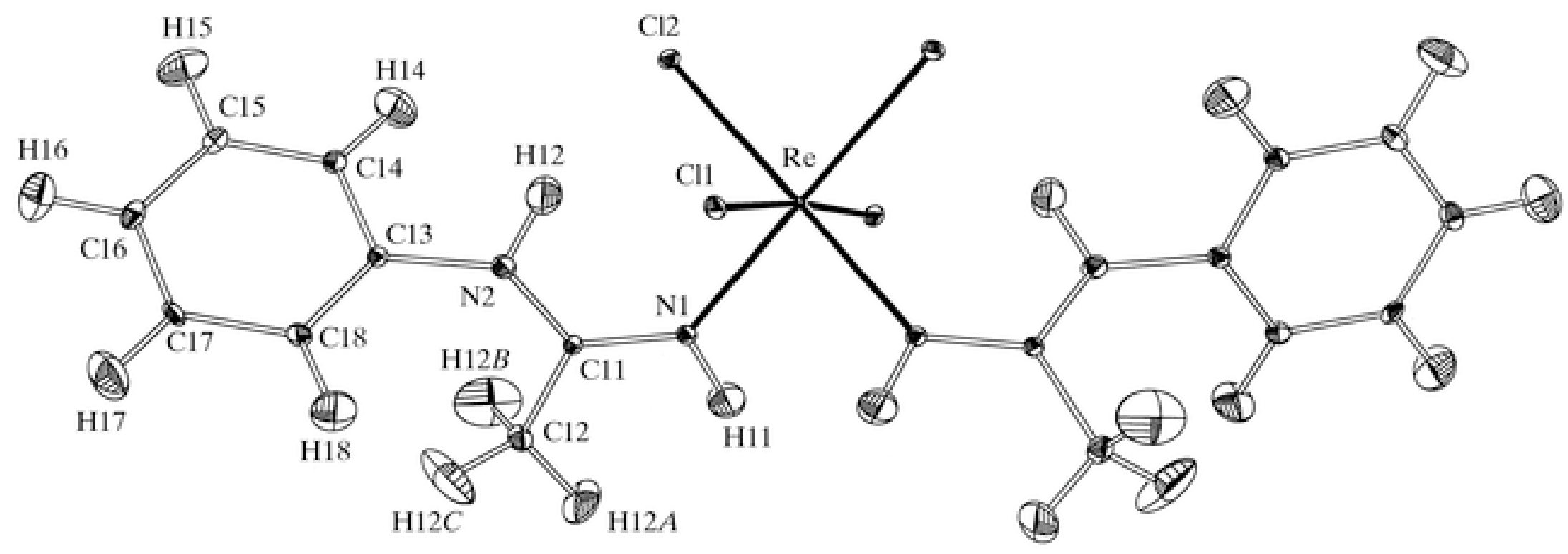

The crystal structure of $\left[\operatorname{ReCl}_{4}\left(\mathrm{C}_{8} \mathrm{H}_{10} \mathrm{~N}_{2}\right)_{2}\right]$ at $11 \mathrm{~K}$ from X-ray data.

Displacement ellipsoids are shown at the $75 \%$ probability level.

(Figgis et al., Acta Cryst. 2001, C57, 1151-1153.) 


\section{Spacing of atoms in crystals is on an Ångstrom scale}

- The wavelength of light that will be diffracted depends upon the spacing of the objects doing the diffracting. Since the spacing of atoms is on an Ångstrom scale, light of similar wavelength-X-ray wavelengths -is diffracted by crystals

- $1 \AA=10^{-10} \mathrm{~m}=0.1 \mathrm{~nm}=100 \mathrm{pm}$

» Light that has wavelengths of 1 or 2 Ångstroms is in the X-ray part of the electromagnetic spectrum

- W. L. Bragg determined the structure of $\mathrm{NaCl}, \mathrm{CsCl}$ and $\mathrm{ZnS}$ by analyzing the X-ray diffraction pattern from single crystals of these inorganic compounds 


\section{The crystal lattice}

- Composed of identical lattice points

- A unit cell is chosen which represents the full symmetry of the lattice

- The crystal is generated by translating the unit cell in three dimensions

- If there is more than one identical molecule within the unit cell, the molecules are related by symmetry operations such as inversion center, mirror planes, rotation axes 


\section{Diffraction}

- Results from interference of waves

"Constructive interference is described by

$\checkmark$ Bragg's Law: $n \lambda=2 d \sin \theta$

$\checkmark \lambda$ is the wavelength of the $X$ rays

$\checkmark d$ is the distance between the crystal planes $\mathrm{h}, \mathrm{k}, \mathrm{I}$

$\checkmark \theta$ is the diffraction angle

"Destructive interference at other angles

- $X$ rays diffracted by the electron density in a threedimensional array (a solid crystal) produces a pattern of spots called the diffraction pattern 


\section{Real vs. Reciprocal space}

- Real space

" the crystal lattice: $a, b, c, \alpha, \beta, \gamma$

" atoms and molecules-electron density

" imaginary $h, k, l$ planes in the crystal spaced distance $d$ apart

- Reciprocal space

» the diffraction pattern-diffraction spots-at angle theta $(\theta)$

- The spacing of the diffraction pattern is inversely proportional to the spacing in the crystal, as we would predict from Bragg's Law: $n \lambda=2 d \sin \theta$ 


\section{Observing the diffraction pattern allows us to deduce the structure}

- Spacing of diffraction spots $\Rightarrow$ size and shape of unit cell

- Systematic absences of spots $\Rightarrow$ symmetry of unit cell

- Spot profile $\Rightarrow$ thermal vibration of atoms

- Spot intensities $\Rightarrow$ atomic types and positions ( $x, y, z$ coordinates)

- Atomic positions $\Rightarrow$

" bond lengths

" bond angles

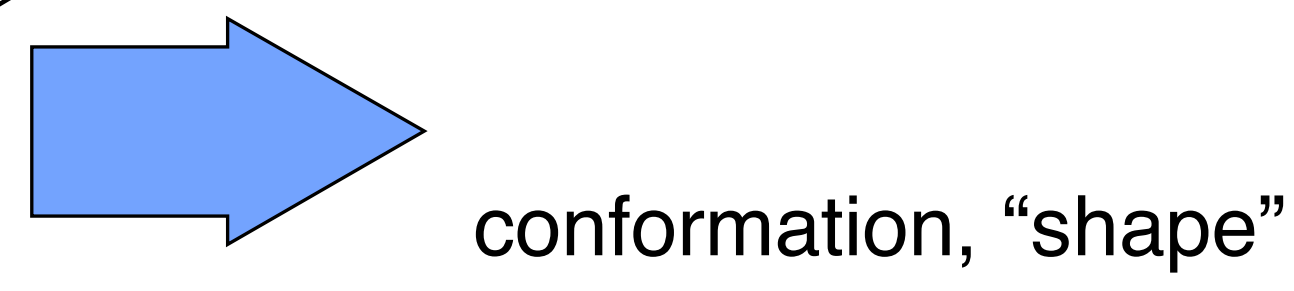

" torsion angles 


\section{A mathematical lens}

- Each set of planes in real space produces one spot in the diffraction pattern

- The spots are NOT the image of the molecule/atoms

- Unfortunately, we have no physical lens to focus the diffracted rays

- Therefore, our "lens" must be a mathematical device, the Fourier transform 


\section{Observed diffraction pattern}

- We observe the intensities of the spots $I_{h, k, l}$ from each $\mathrm{h}, \mathrm{k}, \mathrm{l}$ plane in the diffraction pattern (reciprocal space)

$$
\text { 》 } F_{h k l}=\sum f_{j} e^{2 \pi i(h x+k y+l z)}
$$

$\checkmark$ Summation over all the atoms in the unit cell $\checkmark$ xyz refers to atomic positions (if we knew them) $\checkmark$ structure factor $F_{\mathrm{hkl}}=\sqrt{I_{h, k, l}}$

$\checkmark \mathrm{f}_{\mathrm{j}}$ is the atomic scattering factor for each type of atom; its magnitude depends upon $\mathbf{Z}$

- The intensity of a single spot in the diffraction pattern depends upon the constructive/destructive interference of the diffracted waves from every atom in the crystal! 


\section{Calculated electron density}

- The goal of an X-ray determination is to calculate an electron density map (in crystal space)

» $\rho(x y z)=1 / N \sum F_{h k l} e^{-2 \pi i(h x+k y+l z)}$

$\checkmark$ Summation over all reflections $\mathrm{h}, \mathrm{k}, \mathrm{I}$

$\checkmark \rho(\mathrm{xyz})$ is the electron density

$\checkmark$ xyz refers to an arbitrary grid point in the unit cell

$\checkmark \mathrm{F}_{\mathrm{h}, \mathrm{k}, \mathrm{l}}$ is the structure factor, the square root of the intensity of the diffraction spot

- Atoms must be located at the "peaks" of electron density! 


\section{The two equations are Fourier Transforms of each other}

- The structure factor is the square root of the observed intensities from "diffraction space" (reciprocal space)

- The electron density at a point is a "real space" property

- The FT is a mathematical transform between diffraction space and real space.

- A problem:

" To obtain the electron density, we need to know the value of the structure factors

" To know the structure factor, we need to know the position of the atoms 


\section{Phase Problem}

- We don't have the information we need to calculate the electron density map

- We need structure factors, $F_{h k l}$

- We observe the intensities, $I_{\mathrm{hkl}}$

$$
\checkmark \mathrm{I}_{\mathrm{hkl}}=\mathrm{F}_{\mathrm{hkl}}{ }^{2}
$$

- The observed intensity of a diffraction spot is the square of the structure factor

- The numerical value of the structure factor may be positive, negative, or a complex number. We don't know the mathematical phase of the number. 


\section{Solving the phase problem}

- "heavy atom" method (Patterson method) is effective when there are one or two atoms with much larger $f_{j}$ values than the other atoms in the structure

- "direct" methods (probability methods) are used to solve the structures of small molecules

- multiple isomorphous replacement (MIR) for macromolecular structures

" heavy atom derivatives

" anomalous dispersion

- MAD (multi-wavelength anomalous dispersion) 


\section{Synchrotron radiation}

- Intense

» 30-60 seconds instead of 30-60 minutes for a "frame" of diffraction data

- Tunable

" multiple wavelengths

"stronger anomalous signal at some wavelengths

- Intense radiation, combined with liquid nitrogen temperatures and more sensitive detectors enables larger and larger structures to be solved 


\section{The result of all this work is a model \\ of the molecule}

- ORTEP diagram displays a molecule based upon the atomic positions, showing the thermal ellipsoids that represent the thermal motion of each atom in the molecule.

- A packing diagram shows how the individual molecules pack together in the unit cell of the crystal. As you recall, the molecules in the unit cell are related by symmetry operations.

- A ribbon diagram of a protein doesn't show individual atoms; it represents groups of atoms that are arranged in a repeating motif, such as alpha-helix or beta-sheet. 